\title{
The Design and Realization of fruit Harvesting Robot Based on IOT
}

\author{
Biqing Li", a, Aizhen Zhou ${ }^{2, \text { a }}$, Chongjun Yang, a, Shiyong Zheng, b \\ ${ }^{1}$ College of Mechanical and Electronic Engineering, Hezhou University, Hezhou Guangxi 542899, \\ China; \\ ${ }^{2}$ Liuzhou Jiacheng automotive trim Systems Co. Ltd, Liuzhou Guangxi 545000, China; \\ ${ }^{3}$ College of Computer Science and Information Engineering, Hezhou University, Hezhou Guangxi \\ 542899, China. \\ ajanliful@163.com, b229292710@qq.com
}

\begin{abstract}
As the increasingly developing of computer intelligent industry with automation, intelligent robot has widely been used in various industries. Currently, most of domestic fruits are still picked by manual work. However, manual picking method enhances the fruit farmer's financial costs due to the worker pay raises consistently, and the application of robot in agriculture industry is demanding. Therefore, the intelligent mobile robot picker is launched on the basis of machine visual technology, integrating the mobile carrier, robot arm, picker, traverse mechanism and intelligent module, which achieves the travel route programming of the robot picker, auto-judging the ripe fruit as well as positioning and recognition functions by binocular stereoscopic visual technology.
\end{abstract}

Keywords: IOT (Internet of Things); Fruit harvesting; Binary processing.

\section{Introduction}

A China's fruit industry has been developed rapidly. Its cultivated area and annual output both rank world No.1, and it is the largest fruit planting country. Fruit picking is the most time consuming job requiring majority of the labor input during the whole process. However, the country human labor is decreasing as the migrant worker increases, and it results in labor cost raises, higher expenses, seriously restricting the developing of fruit industry. As to future develop the fruits industry, it requires to control the product cost and reduce manual work. Thus, the wide application of intelligent robot picker in agriculture production it is significant for cost cutting down with improved efficiency [1]. In Japan, America and German, for the shortage of human labor, intelligent robot in agriculture is extensively adopted. As the leading fruit production country, China turns its attentions on the mechanical picking; in this way, the China's fruit industry would be largely promoted. This article designs the intelligent fruit robot picker on the basis of fruit planting environment and actual pickingprocess as well as the machine visual technology's research on fruit picking- process to achieve the intelligent picking- method in agriculture production.

\section{Structure Design}

This robot is constituted by mechanical section and electrical intelligent control system. The mechanical section includes the mobile carrier, robot arm, picker, traverse slider, and the electrical intelligent control system mainly consists of control computer, servo motor drive, double CCD camera, sensor control module, data acquisition card, GPIB card, motion card, Lithium battery power box, GPIB card and control system[2].

The mechanical section is the hardware platform for intelligent robot picker, and it formulates the solid foundation for ripe fruit recognition and picking of the robot. It achieves robot picker auto control by joint in each component with different functions. The mobile carrier is the basis of intelligent robot, and it is also the platform for other controlling acquisition systems. The signal acquisition, motor drive, sensor module as well as intelligent control system of the intelligent robot picker demand the mobile carrier, and it adopts crawler travelling method to adapt to the orchard ground, with large contacting surface and large stressed area on the ground, and gives less pressure to it. 
The premise of the robot picker's performance is to recognize the ripe fruit with three-dimensional positioning at first, largely improved the picking efficiency. The picker of robot relies on arm-around method as human being during the process, which is depended on the three-dimensional positioning. The three-dimensional visual technology shots the images from different angles by two cameras, and then transmits them to the control computer, through to obtain fruit three-dimensional image in comparison of the different angles. Next, the intelligent robot picker starts picking according to the image [3].

The machine visual system mainly consists of control computer, LED light, special optical lens, CCD camera and digital image processing module. When working, the system transfers the to-be-tested target to digital image signals over the double CCD camera, and then transmits it to the PC machine, it would collect each fruit characteristics by multiple calculations in accordance to the its color over the special image processing software. At last, it makes accurate judgement from the setting value and other conditions. The identification segmentation and positioning technologies of the robot picker include target fruit segmentation recognition, ripeness judgement and target fruit's spatial coordinates calculation.

\section{Target Fruit Segmentation Recognition and Ripeness Judgement}

At first, it requires correct segmentation recognition and ripeness judgment to the target fruit for the correct picking, thus the robot picker could substitute for manual picking by human labor. The critical task of recognition is to collect the fruit image samples, which relies on CCD camera shooting in accordance to the completed fruit samples by image information. Next is to set up the color model, to it divides the fruit and surrounding environment by segmentation technology, and then recognizes the fruit. Furthermore, it judges the ripeness of target fruit by comparing the tricolor calculations. The tricolor model RGB is shown as fig.1.

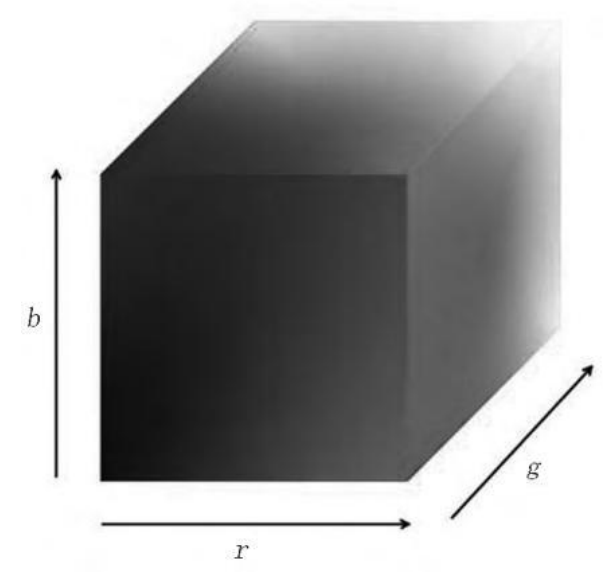

Fig. 1 RGB color model

By the tricolor RGB, it could successfully divide the target fruit. In addition, the image processing module compares the ripe fruit color sample in PC machine database and judges its ripeness based on its color.

\section{Spatial Coordinate Calculation of Target Fruit}

By human being, they could grab the target object eventually via the distance feedback by eyes with consistent judgement during approaching of target, while the robot picker doesn't possess the human mind and feedback ability, and it requires the sensor and control system to simulate the human processing method. During fruit picking [4], it obtains the whole fruit tree's information via the CCD camera, and then transfers to the image processing system. Furthermore, it recognizes the target fruit by tricolor RGB segmentation, and accurately locates the target fruit position relating to the spatial coordinate by the infrared source, fruit outline as well as image differences simultaneously captured by the two cameras [5]. 


\section{Route Recognition Programming of Intelligent Robot Picker}

The intelligent robot picker has to move all around in the orchard, which is demanding to detect the barrier as to avoid it. Therefore, it needs to program the route recognition to avoid the barrier, thus to complete the picking task.

\section{Conclusion}

It devices the intelligent mobile fruit robot picker based on machine visual technology in accordance to the harvest requirement of ripe fruit from the farmer. With integration of machine visual technology and automation technology, this robot features simple mechanical structure, flexible coordination, swift movement which could avoid the barrier successfully with fast target recognition as well as high proficiency. It full fills the mechanical intelligence, and automation unmanned processing for agriculture picking work, which has the promising future.

\section{Acknowledgements}

This work is supported the following fund:

2016 The project of improving the basic ability of young teachers in Colleges and universities in Guangxi: "Design and development of electronic commerce platform of agricultural products based on Semantic Technology" (No, KY2016YB455).

2016 Guangxi higher education teaching reform project: "Exploration on the cultivation mechanism of the teaching characteristics of Communication Engineering Specialty Based on ZTE ICT education platform"

2015 college students' innovative training program: "Research on the application of value added travel experience in the mobile terminal of the "ethnic custom travel' in Guangxi" (No 201511838070); \&\& “The design and development HeYuanTong Campus Mobile Phone APP based on Android" (No 201511838034).

2015 Teaching case project construction project of hezhou university: "Tourist positioning and guiding system project of Huangyao scenic"; \&\& "Development and application of the information management system of 'Huang Yao Tong"'.

Project of scientific research and technology development project of Hezhou: "Design and implementation of agricultural products e-commerce platform based on Semantic Technology" (No, Hekeneng 1506006)

Scientific research project of hezhou university: "Research and development of E-government platform based on mobile terminal" (No 2015ZZZK03);

Reform in Education project of hezhou university: "Internet of things" (No hzxytszy201501); "Research on application personnel training model of Communications for SMEs" (No hzxyjg201525);

Master degree discipline construction scientific research and Cultivation Project: "Research on the social work service of the teenagers' Network Addiction" (No 2015SHGZ005) \& \& "Research on the intelligent development of rural tourism in Guangxi based on the Internet of things" (No 2015MTA16).

\section{References}

[1] Zheng SQ, DZ; Qiang, Z; Li, BQ. Design and Implementation of Supermarket Personnel Management System Based On Java. In: Chen PZ, S, editor. 2nd International Conference on Education, Management and Computing Technology (ICEMCT); Tianjin, PEOPLES R CHINA: ATLANTIS PRESS; 2015. p. 1724-7. 
[2] Li BG, WL; Zheng, SY; Yue, XG. OPTIMISATION DESIGN OF CORN PRECISION SEEDER BASED ON MULTI-ROUTE AND MULTI-CHANNEL CONTROL [J]. JOURNAL OF THE BALKAN TRIBOLOGICAL ASSOCIATION. 2015, 21(4A): 1215-23.

[3] Zheng SG, Weili; Li, Biqing. Social Work in Teen Addiction Correction Services Research under the New Situation. In: Wang JX, P, editor. 4th National Conference on Electrical, Electronics and Computer Engineering (NCEECE); Xian, PEOPLES R CHINA: ATLANTIS PRESS; 2016. p. 252-5.

[4] .Zheng SG, WL; Li, BQ. Digital Display Design of Ethnic Clothing of Nanling. In: Liang ZL, X, editor. 4th International Conference on Mechatronics, Materials, Chemistry and Computer Engineering (ICMMCCE); Xian, PEOPLES R CHINA: ATLANTIS PRESS; 2015. p. 2805-8.

[5] Shiyong Zheng WG, Jing Wang, Biqing Li, Deze QIN. Analysis of Internet of Things Talent Training and Curriculum System Innovation. 3th International Conference on Education, Management and Computing Technology; HangZhou, China: ATLANTIS PRESS; 2016. p. 957-60. 